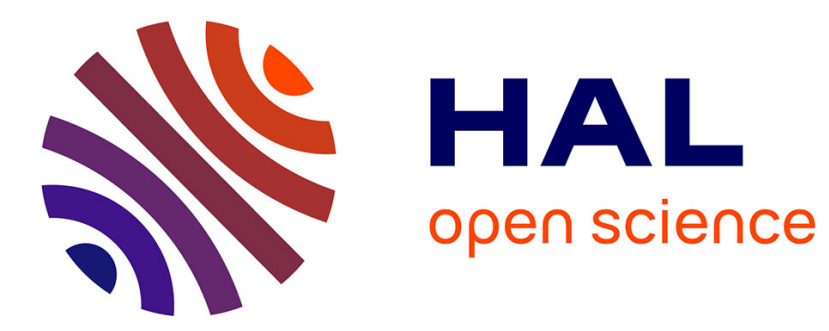

\title{
Le harcèlement chez les adolescents victimes : méthodologie d'une recherche internationale
}

Marjorie Roques, Deise Matos Do Amparo, Neslihan Zabci, Didier Drieu, Teresa Rebelo, Florent Cosseron

\section{- To cite this version:}

Marjorie Roques, Deise Matos Do Amparo, Neslihan Zabci, Didier Drieu, Teresa Rebelo, et al.. Le harcèlement chez les adolescents victimes : méthodologie d'une recherche internationale. Annales MédicoPsychologiques, Revue Psychiatrique, 2019, 177 (10), pp.999-1004. 10.1016/j.amp.2019.09.007 hal02479882

\section{HAL Id: hal-02479882 \\ https://hal.science/hal-02479882}

Submitted on 21 Dec 2021

HAL is a multi-disciplinary open access archive for the deposit and dissemination of scientific research documents, whether they are published or not. The documents may come from teaching and research institutions in France or abroad, or from public or private research centers.
L'archive ouverte pluridisciplinaire HAL, est destinée au dépôt et à la diffusion de documents scientifiques de niveau recherche, publiés ou non, émanant des établissements d'enseignement et de recherche français ou étrangers, des laboratoires publics ou privés.

\section{(ㄷ)(1) $\$$}

Distributed under a Creative Commons Attribution - NonCommerciall 4.0 International 


\title{
Communication
}

Le harcèlement chez les adolescents victimes : méthodologie d'une recherche internationale

Adolescents victims of bullying. Presentation of a methodology for an international research

\author{
Marjorie Roques a , Deise Matos Do Amparo ${ }^{b}$, Neslihan Zabci ${ }^{c}$, Didier Drieu ${ }^{d}$, Teresa \\ Rebelo $^{\text {e }}$, Florent Cosseron ${ }^{\mathrm{f}}$
}

a) Université de Normandie, Esplanade de la Paix14032 Caen Cedex 5.

Maître de conférences des universités en psychologie, Membre du LPCN (EA7452) Université de Caen Normandie, Psychologue clinicienne, Unité de crise et d'hospitalisation pour adolescents Service de psychiatrie de l'enfant et de l'adolescent, CHU de Caen, France.

b) Professeur des universités en psychologie, Université de Brasilia, Brésil.

c) Maître de conférences des universités en psychologie, Université de Maltèpe, Turquie.

d) Professeur des universités en psychologie, CRFDP, Université de Rouen.1, rue Thomas Becket, 76130 Mont-Saint-Aignan, France. Psychologue clinicien, ACSEA, Caen. 1, impasse des Ornes, 14200 Hérouville Saint-Clair, France.

e) Maître de conférences des universités en psychologie, CRFDP, Université de Rouen. 1, rue Thomas Becket, 76130 Mont-Saint-Aignan, France.

f) Pédopsychiatre, Clinique Château du Bel Air, Paris, ACSEA. 35 Rue Albert Thomas, 91560 Crosne, France.

Auteur correspondant : Marjorie Roques, Service de psychiatrie de l'enfant et de l'adolescent du CHU de Caen, 14, avenue Georges Clémenceau, 14033 Caen, France.

Tél. :06 88813851

Email : marjorie.roques@unicaen.fr

\section{Résumé}

Le harcèlement est un phénomène complexe et mondial. De 100 à 600 millions d'adolescents seraient concernés dans le monde. De nombreuses études se sont penchées sur ce phénomène 
ces vingt dernières années, mais les publications portant sur des recherches françaises sont rares. Les deux objectifs de cet article sont :

- d'apporter des précisions concernant la définition du harcèlement afin de circonscrire le cadre de notre recherche. Outre les quatre facteurs habituellement cités dans les études, la fréquence, l'intensité, la répétition et l'asymétrie, nous avons postulé que la définition du harcèlement à l'adolescence ne pouvait se départir d'autres éléments tels que l'adolescence et le groupe. Quant à son repérage clinique, des signes de souffrance psychique aiguë ayant trait au traumatisme et aux traces d'une relation d'emprise, retrouvés dans toute violence psychologique, peuvent être relevés ;

- de présenter la méthodologie d'une recherche internationale à méthodologie mixte (outils qualitatifs entretiens, épreuves projectives et outils à méthodologie quantitative : questionnaires) actuellement en cours.

À l'appui d'une revue de la littérature internationale, de notre pratique clinique et des premières données analysées (non encore publiées), nous en avons déduit que la complexité du harcèlement tenait en la superposition de vulnérabilités psychologiques et familiales. Pour conclure, nous avons proposé un dispositif de prise en charge spécifique de prévention secondaire (théâtre-forum et groupe multifamilles).

Mots clés : Adolescent ; Famille ; Harcèlement moral ; Psychologie ; Recherche ; Revue de la littérature ; Syndrome post-traumatique ; Victime ; Violence ; Vulnérabilité

\footnotetext{
Abstract

Bullying is a complex and global phenomenon. It is estimated that 100 to 600 million adolescents are affected worldwide. Many studies have examined bullying over the past twenty years, but French publications remain rare.

Objectives. - The objectives of this article are to clarify the definition of bullying in order to circumscribe the framework of our qualitative research, and to present the methodology of an international research currently underway.

Patients and methods. - Our sample consists of 40 ou 50 French, Brazilian and Turkish adolescents. These patients have the following characteristics:

- they are aged 12 to 18 years;

- they are consultants in specialized centers;

- they are interviewed at least three months after bullying has stopped, for ethical reasons and in order to evaluate the PTSD according to international classifications;
} 
- finally, they show clinical signs related of psychological suffering to the trauma and traces of a controlling relationship.

The criteria for non-inclusion are as follows: severe psychiatric disorders (active delusional pathologies), bullying still ongoing. In order to test our first hypothesis regarding the study of psychological and family vulnerabilities, we chose several tools: the non-directive research interview and the projective tests Rorschach, TAT, family drawing and house drawing. In order to test our second hypothesis regarding the consequences of bullying, namely the study of symptoms of post-traumatic stress, we chose three tools: the non-directive research interview, the TraumaQ and the SCL-90.

Results. - Following a review of the literature and in support of our clinical practice and the initial data from this research, we discussed complementary criteria for defining bullying, in addition to those usually mentioned, in other words: repetition, intensity, frequency and the power of imbalance. Bullying, as a violent relational process, includes clinical signs of acute psychological suffering that often reveal the presence of symptoms of post-traumatic stress and traces of control mechanisms in the victim that are found in any emotional abuse. We have also focused our definition on two essential dimensions: adolescence and the group, and more particularly on taking into account adolescence as a time of narcissistic fragility and the reactivation of psychological problems, which often make it necessary to support a group of peers. This support appears all the more "vital" as these young people move away from their family group at the same time. In these circumstances, some adolescents, who are identified as having family and psychological vulnerabilities and who have difficulty positioning themselves in a group, may become "easy" prey and be subjected to violence. Without considering a typical bullied profile, the question of psychological vulnerabilities and in particular trauma, i.e. the appetite for repeated trauma and overexposure to potentially traumatic situations, is an interesting avenue.

Conclusion. - The complexity of bullying lies in the superposition of family and individual factors. Like any violence, it has long-term effects, and it seems essential to offer specific care that makes it possible, in addition to alleviating suffering by providing support, to carry out a thorough analysis of the psychological issues underlying the establishment of a controlling relationship and its sustainability. The secondary prevention component, which includes specific care beyond primary prevention programs in schools, therefore seems important to us to develop. At the end of this article, we will detail the care support which combines the forum theatre method and a multi-family group that we suggest to the victims of bullying. 
Key-words: Adolescent; Bullying; Family; Literature paper; Psychology; Post-traumatic syndrome; Research; Victim; Violence; Vulnerability

\section{1. État des lieux de la question}

Le harcèlement est un phénomène mondial. De 100 à 600 millions d'adolescents seraient concernés dans le monde [1]. De nombreuses études se sont penchées sur le phénomène ces vingt dernières années. D'ailleurs, les publications, majoritairement en langues espagnole et anglaise [2] ont triplé entre les années 1980 et 2007 [3].

Dans la mesure où le harcèlement se manifeste dans l'institution scolaire mais aussi en dehors de l'école, réalisant une emprise continue via le cyberharcèlement, c'est-à-dire sans moment de répit, nous emploierons, dans cet article, le terme de harcèlement en dépit de nombreuses similitudes avec le harcèlement dit «scolaire » [4]. À l'appui d'une revue de la littérature internationale sur la question, nous avons tout d'abord tenté de délimiter ce processus afin de circonscrire le cadre de notre recherche.

La principale difficulté à laquelle nous avons été confrontés au départ de notre étude a été de fournir une définition du harcèlement qui échappe à tout consensus. En effet, comment étudier de manière rigoureuse un phénomène que l'on ne parvient pas à cerner et qui ne relève pas de critères objectifs? Autrement dit, comment savoir si un jeune est victime de harcèlement? Si nous souscrivons à l'idée de la plupart des auteurs selon laquelle les quatre facteurs habituellement cités dans la revue de la littérature - autrement dit : la fréquence, l'intensité, la répétition et la durée - constituent des piliers de ce processus violent, il nous semble cependant que d'autres éléments entrent en jeu. Aussi avons-nous fait le choix de rester au plus près de ce que nous enseigne notre clinique. En écho avec notre pratique auprès de cette population, nous proposons de compiler plusieurs définitions d'auteurs ayant étudié spécifiquement le harcèlement chez les adolescents, avec d'autres qui ont travaillé sur la violence et les mécanismes d'emprise afin d'apporter une définition la plus précise possible du harcèlement à l'adolescence.

\section{Définition du harcèlement}

Le harcèlement se définit comme un ensemble d'agressions psychologiques et/ou 
physiques répétitives plus ou moins subtiles et directes qui se répètent et durent dans le temps. Dans ce processus, sont retrouvés les mêmes mécanismes psychologiques que dans toute relation d'emprise et des ressentis délétères chez la victime (honte, culpabilité, sentiment d'humiliation). Le sens d'emprise vient du domaine juridique et renvoie à la mainmise administrative sur une propriété privée. En psychanalyse, Roger Dorey [5] situe le désir d'emprise «au cœur de la relation intersubjective » consistant en « une atteinte portée à l'autre en tant que sujet désirant ». Il distingue l'emprise perverse, qui vise l'appropriation de l'autre, de l'emprise obsessionnelle qui vise la destruction du désir de l'autre et «dans laquelle celui qui en use par la force essentiellement, est en position de pouvoir sur l'autre ». Nous retrouvons en cela les caractéristiques du harceleur qui vise à disqualifier sa victime, à la discréditer, à l'isoler et à la brimer, générant des représentations négatives chez elle, qu'elle finit par faire siennes. D'ailleurs, la victime finit très souvent par intégrer l'idée qu'elle mérite cette situation. Ce procédé, que Sandor Ferenczi [6] avait décrit dans les cas de violences sexuelles, et qu'il nomme identification à l'agresseur, rend compte d'un fantasme de punition que le sujet intériorise face à la violence de son agresseur. À partir du moment où l'agresseur est devenu intrapsychique, il perd son statut d'objet différencié pour devenir une partie du moi de la victime. On constate, à ce moment précis, un brouillage des repères : entre soi et l'autre, entre Moi et non-Moi, entre réalité externe et réalité psychique.

Concernant la communication à l'œuvre dans la relation harceleur/harcelé, elle semble au service d'une certaine perversion du lien. Soit un discours unilatéral et froid est instauré et génère un climat de terreur, de peur, soit les victimes de harcèlement subissent le silence et l'indifférence, ou encore, les échanges peuvent être basés sur l'émission de messages paradoxaux qui déstabilisent la victime au point d'induire chez elle une confusion des registres, confusion qui peut être renforcée par le harceleur via divers moyens comme l'ironie par exemple. C'est alors que le sujet harcelé doute de l'authenticité de son vécu affectif, voire de la réalité des faits.

Afin d'aller plus loin encore dans notre définition, nous prendrons en considération l'idée que souvent, harceleur et harcelé ont été amis ou partenaires amoureux par le passé [7] [4]. Dans ce contexte, le harceleur a un ascendant plus marqué encore sur sa victime, ce qui assoit son pouvoir et accroît l'asymétrie, accentuant ainsi la souffrance de la victime. Ajoutons à cela le rôle des témoins, actifs ou passifs du harcèlement (lorsqu'ils ne dénoncent pas les faits) qui valident, d'une certaine manière, le procédé du harceleur. Parfois malgré eux, ils privent de façon radicale la victime de soutien et d'empathie, accentuant son ostracisme et son isolement. Accablée par le phénomène de groupe, sa souffrance psychique 
n'en est que plus augmentée. La victime de harcèlement, étant exposée intensément et de manière continue à des violences répétées, apparaît comme particulièrement tourmentée. Dans nos travaux antérieurs, nous avons pris le parti de dire que le harcèlement spécifiquement adolescent mérite d'être lu sous un double prisme [8]. D'une part, sous celui de l'adolescence car le harcèlement atteindrait son niveau maximal lors de cette période de grande vulnérabilité relationnelle et émotionnelle [9], période par ailleurs propice à tous types de passages à l'acte et de symptômes du fait, notamment, de la maturité sexuelle [10-12]. Certains chercheurs vont même jusqu'à qualifier le harcèlement à l'adolescence de stratégie adaptative spécifique de cette période qui permettrait d'aménager les relations amoureuses [13] et de mettre à distance la sexualité [14], vécue comme angoissante.

Outre le prisme de l'adolescence, nous nous sommes intéressés à celui du groupe, plus précisément familial, et par extension, le groupe de pairs, qui participe pleinement à notre compréhension du harcèlement psychologique à l'adolescence ayant lieu en groupe. Nous nous sommes d'abord demandé si les jeunes concernés par le harcèlement n'auraient pas engrammé préalablement, c'est-à-dire dans les échanges au sein de leur propre famille, une perception inadéquate de la relation, le harcèlement constituant ainsi l'unique modalité relationnelle possible, via l'évitement de l'autre ou, à son extrême opposé, l'agression de l'autre. Concernant le groupe extra-familial, il peut, dans le meilleur des cas, représenter un espace sécure et une voie de valorisation narcissique, dans la mesure où l'affiliation à un groupe de pairs joue un rôle majeur dans sa construction identitaire de l'adolescent, il peut aussi avoir des effets néfastes jusqu'à être garant de l'existence même de certains jeunes vulnérables [15]. Dans ce contexte, l'adolescent vulnérable peut être exposé à tous types de risques, notamment à celui de devenir la cible d'un autre jeune ou à celui de développer des troubles psychiques à l'âge adulte [16].

\section{Problématique et hypothèses}

À l'issue d'une revue de la littérature internationale et à l'appui de notre pratique clinique, nous avons émis deux hypothèses qui visent à explorer les vulnérabilités chez les adolescents victimes et les conséquences du harcèlement psychologique observables chez ces jeunes.

Notre première hypothèse s'appuie sur l'idée que s'il n'existe pas de personnalité ou de profil type de harcelé, le harcèlement ne s'adresse pas à n'importe quel jeune et rendrait compte de vulnérabilités croisées, individuelles et groupales, susceptibles de favoriser 
l'émergence de ce processus. Nous postulons une superposition de facteurs familiaux sur des fragilités psychiques préexistantes (et qui seraient, de manière circulaire, le fait même de ces facteurs). En outre, la quête d'autonomie propre à l'adolescence suppose un sentiment de sécurité interne dont certains adolescents vulnérables ne disposent pas, pour supporter la séparation et les affects qui l'accompagnent. Autrement dit, comment exporter dans le groupe de pairs ce qui n'est pas solide en soi, ni chez soi ? En effet, si l'insécurité intérieure est majorée par la poussée pulsionnelle de la puberté et l'autonomisation propres à l'adolescence, il n'en reste pas moins qu'elle reste étroitement liée à l'environnement dans lequel l'adolescent a évolué et à la place qu'il occupe réellement ou imaginairement dans sa propre famille.

Notre deuxième hypothèse repose sur l'idée selon laquelle, chez les jeunes ayant été harcelés, il est possible de retrouver les mêmes symptômes que ceux du syndrome de stress post-traumatique (troubles du sommeil, angoisse, idées suicidaires etc.) et ce, à distance de l'événement (plusieurs mois voire plusieurs années). Cette idée avait déjà été abordée dans deux autres études qui avaient rapporté un niveau élevé de stress post-traumatique. Mais ce type de recherches reste rare [17].

Ces éléments pré-cités, de même que la définition du harcèlement comme violence, nous indiquent tout l'enjeu d'identifier le harcèlement comme traumatisme, ayant des effets à long terme, ainsi que la nécessité d'une prise en charge de toute urgence pour ces adolescents, en deçà des programmes de prévention dans les établissements scolaires. En effet, la plupart des recherches se centrent sur le volet de la prévention primaire, certes intéressante mais insuffisante. Après une évaluation des vulnérabilités individuelles et familiales et des conséquences psychologiques du harcèlement, notre recherche comporte un deuxième temps portant sur la prévention secondaire, c'est-à-dire sur les dispositifs thérapeutiques proposés à ces adolescents.

\section{Méthodologie d'une étude se déroulant sur 48 mois}

Ce sont sur ces points de repérage des vulnérabilités dans un premier temps, et d'évaluation des prises en charge spécifiques dans un second temps, via un protocole à méthodologie double (qualitative et quantitative), que repose notre recherche. L'inclusion des patients dans la recherche se fait du temps 0 jusqu'à 18 mois pour l'évaluation des vulnérabilités psychologiques et familiales. Puis, ces patients sont intégrés aux prises en charge thérapeutiques de 18 à 36 mois. 
Concernant les critères d'inclusion, les jeunes patients présentent les caractéristiques suivantes:

- $\quad$ âge : < 12 à 17 ans >, sortis de la période de latence ;

- $\quad$ rencontrés au moins trois mois après que le harcèlement a cessé (évaluation du PTSD, respectivement aux critères des classifications internationales, DSM-5) ;

- étant donné qu'il s'agit d'une population sensible, ces adolescents sont obligatoirement consultants dans des services spécialisés ;

- $\quad$ outre le discours rapporté par le patient lui-même, les parents, les professionnels de collèges et de lycées, les traces d'un cyberharcèlement, ces jeunes présentent des signes cliniques de souffrance psychique ayant trait aux traces du traumatisme et d'une relation d'emprise.

Concernant les critères de non-inclusion, les jeunes patients ne doivent pas présenter les caractéristiques suivantes :

- $\quad$ troubles psychiatriques sévères (pathologies délirantes actives) ;

- $\quad$ harcèlement en cours.

La recherche se divise en deux temps.

Un premier temps de 0 à 18 mois est consacré à l'inclusion des patients dans la recherche et à l'évaluation des vulnérabilités psychologiques et familiales et des conséquences du harcèlement psychologique.

Ce premier temps est partagé en deux :

a) Étude des vulnérabilités psychologiques du jeune qui comprend l'utilisation des outils suivants :

un premier entretien non directif de recherche pour recueillir des données biographiques ;

- des épreuves projectives Rorschach et TAT, reconnus mondialement reconnus pour leurs qualités diagnostiques. En outre, ces outils présentent l'avantage de saisir, sur un temps court, des problématiques psychologiques autrement peu accessibles [18].

b) Étude des vulnérabilités familiales: Étant donné que les adolescents sont souvent réticents aux questionnaires ou aux entretiens, nous avons choisi le dessin pour accéder, par cette médiation, aux représentations qu'ils ont de leur propre famille, un sujet particulièrement sensible à cette période de la vie. Le dessin de la famille [19,20] et le dessin de la maison [21] consistent à demander à l'adolescent de dessiner respectivement sa famille 
puis une famille et sa maison, et enfin de compléter par un commentaire sur ces dessins. Le choix du dessin dans le cadre de notre étude est stimulé par divers motifs. Tout d'abord, nous pensons que le dessin, utilisé comme une épreuve projective, représente le moyen le moins frontal par l'intermédiaire duquel l'adolescent peut communiquer son vécu, son ressenti et ses désirs en rapport avec sa famille. En ce sens, nous considérons que le dessin permet d'avoir accès aux enjeux symboliques sous-jacents et à la signification attribuée par le sujet. Le sujet peut aborder ses relations avec l'environnement et ses expériences marquantes. La consigne des dessins axée sur l'imaginaire vise à aborder les enjeux et l'histoire familiaux de manière détournée mais aussi permet de savoir comment l'adolescent investit son «chez-soi » et comment il se protège des autres (exposition au harcèlement); tel est le cas pour le dessin de la maison, via la métaphore de la maison, considérée à la fois comme symbole du milieu familial et comme métaphore corporelle [22]. Pour l'enfant, la maison est le premier espace qu'il explore et où il vivra ses premières expériences. En cela, le dessin de la Maison est chargé en souvenirs et en affects et il existerait une relation forte entre la maison, le corps et le psychisme. Chaque dessin est analysé à partir d'une grille de cotation, appelé «cahier de relevé de signes » pour le dessin de la maison.

c) Étude des conséquences du harcèlement :

* Un entretien clinique de recherche pour recueillir les éléments concernant le vécu du harcèlement psychologique. L'entretien clinique de recherche est une méthode qualitative de recueil de données [23] [24] qui permet d'accéder à des informations subjectives (histoire de vie, représentations, sentiments, affects, expérience) témoignant de la singularité et de la complexité d'un sujet. L'analyse du contenu de l'entretien fait l'objet d'un découpage et d'une analyse thématiques [25] effectué suite à une lecture d'ensemble.

*Un hétéro-questionnaire TraumaQ [26] qui rend compte des symptômes du traumatisme, de l'insertion sociale et de la qualité de vie. L'élaboration de ce questionnaire s'appuie sur deux approches principales du traumatisme: d'une part, l'approche symptomatique du DSM, d'autre part, une approche holistique en référence à la psychanalyse et notamment, à la névrose traumatique. Ce questionnaire n'évalue pas le stress, il évalue le syndrome posttraumatique (troubles traumatiques aigus et chroniques) voire des modifications de la personnalité suite à un événement traumatique. Plutôt que d'employer le terme de syndrome post-traumatique, les auteurs préfèrent parler de traumatisme psychique afin de ne pas réduire leur exploration aux symptômes pathognomoniques (répétition, évitement phobique, symptômes neurovégétatifs) et d'ouvrir à une analyse du «vécu traumatique » (honte, 
culpabilité, agressivité, répercussions sur la qualité de vie). La première partie du questionnaire composée de dix échelles ${ }^{1}$ est divisée en deux séquences temporelles : pendant l'événement, depuis l'événement. La deuxième partie du questionnaire est facultative et concerne le délai d'apparition et la durée des troubles décrits. À l'issue de l'administration et de la cotation du questionnaire, une analyse typologique, comprenant quatre profils ${ }^{2}$ peut être établie. Au regard de notre revue de la littérature, ce questionnaire se prête tout particulièrement à notre population et à la thématique de notre étude, pour venir conforter ou non l'hypothèse d'un traumatisme suite au harcèlement, tant en termes de signes que de vécu traumatique et l'idée d'une prise en charge.

* Un auto-questionnaire SCL-90 [27] qui évalue la détresse psychologique et le profil psychopathologique à partir de neuf échelles symptomatiques. «Le SCL-90-R est un inventaire d'auto-évaluation de symptômes conçu pour refléter les profils psychologiques de sujets sains, ou présentant une pathologie organique ou psychiatrique » (p. 5) «le SCL-90-R est une mesure de l'état actuel, à ce moment précis, de la symptomatologie psychologique. Il n'est pas une mesure directe de la personnalité » (p. 5). Ce questionnaire est coté et interprété selon deux échelles symptomatiques ${ }^{3}$ et trois indices généraux de détresse ${ }^{4}$. Cet outil se présente sous la forme d'une liste de problèmes que les gens rencontrent parfois, le sujet doit entourer la réponse qui traduit à quel point ce problème l'a gêné ou perturbé ${ }^{5}$ au cours des sept derniers jours, y compris aujourd'hui.

Le second temps, de 18-48 mois, est consacré à l'inclusion des patients de la première phase dans le second temps de cette recherche, c'est-à-dire dans des dispositifs de prise en charge

\footnotetext{
1 1. Pendant l'événement: Échelle A (8 items) les réactions immédiates, physiques et psychique pendant l'événement

2. Depuis l'événement : Échelle B (4 items) : le symptôme pathognomonique de répétition : les reviviscences, l'impression de revivre l'événement, les flashes et l'angoisse attachée à ces répétitions ; Échelle C : (5 items) les troubles du sommeil ; Échelle D (5 items) : l'anxiété, l'état d'insécurité et les évitements phobiques ; Échelle E (6 items) : l'irritabilité, la perte de contrôle, l'hyperviligance et l'hypersensibilité ; Échelle F (5 items) : les réactions psychosomatiques, physiques et les troubles addictifs; Échelle $\mathrm{G}$ (3 items) : les troubles cognitifs (mémoire, concentration, attention); Échelle H (8 items) : les troubles dépressifs (désintérêt général, perte d'énergie et d'enthousiasme, tristesse, lassitude, envies de suicide); Échelle I (7 items) : le vécu traumatique : culpabilité, honte, atteinte de l'estime de soi, sentiments violents et colère, impression d'avoir fondamentalement changé) ; Échelle J (11 items) : la qualité de vie.

2 Profil A : syndrome psycho-traumatique intense ; profil B : syndrome anxio-dépressif (sans syndrome psychotraumatique déclaré); syndrome psycho-traumatique modéré (sans troubles dépressifs); Profil D : syndrome psycho-traumatique léger.

${ }^{3}$ Somatisation, obsession-compulsion, traits sensitifs, dépression, anxiété, hostilité, anxiété phobique, idéations paranoïaques, traits psychotiques.

${ }^{4}$ Indice global de gravité, intensité des symptômes rapportés, nombre de symptômes rapportés.

${ }^{5} 0$ : pas du tout $; 1=$ un peu ; $2=$ modérément $; 3=$ beaucoup $; 4=$ extrêmement.
} 
durant six mois minimum. La prise en charge est plurifocale, individuelle et groupale, impliquant les jeunes, les professionnels du soin et les parents. Ce dispositif combine :

- $\quad$ - un suivi individuel ;

- - un groupe à médiation, reconnu comme étant adapté à la clinique de l'adolescence [28] et plus particulièrement une médiation-théâtre déjà utilisée dans des cas de harcèlement scolaire [29]. Nous avons choisi la méthode du théâtre-forum : approche groupale centrée sur la résolution des conflits. Le théâtre forum est une technique particulière du théâtre de l'opprimé (Augusto Boal). Son principe directeur est de permettre au spectateur de devenir « acteur de sa propre vie » sur la scène du théâtre. La troupe présente un problème (injustice, oppression) non résolu devant un public qui s'identifie à celui-ci. Ensuite se déroule un débat théâtral entre les acteurs et les spectateurs (avec pour meneur de jeu un personnage médiateur), qui vont chercher à résoudre théâtralement le problème initial. Il faut savoir que la méthode du théâtreforum a été régulièrement utilisée pour la prévention des situations de violence chez les jeunes ;

- - un groupe multifamilles animé par un clinicien-chercheur et un pédopsychiatre. En parallèle des groupes de théâtre-forum adressés aux adolescents, nous proposons l'utilisation complémentaire et innovante de la méthode de la thérapie multifamiliale. Cette approche centrée sur le soutien et l'élaboration entre familles concernées par une problématique de vulnérabilité. Nous pensons en effet que l'intervention de tiers adultes professionnels mais aussi parentaux est indispensable pour interrompre le processus de harcèlement. Dans son fonctionnement, la thérapie multifamiliale (TMF) consiste à rassembler dans un contexte thérapeutique plusieurs familles autour d'une difficulté commune. Elle constitue un outil thérapeutique novateur et valorisant pour les familles.

Afin d'évaluer l'efficacité des prises en charge, nous proposons une batterie de tests au début de la prise en charge et à la fin de la prise en charge dans une perspective de test/retest. Cette démarche nous permet d'évaluer l'évolution des symptômes du traumatisme et de mettre en tension les données obtenues à l'entrée du dispositif, avec les données obtenues à la fin de la prise en charge.

\section{Conclusion}

Nous avons souhaité vous présenter la méthodologie d'une recherche internationale sur le harcèlement dont la première partie est en cours de réalisation.

Une revue de la littérature internationale nous a permis de constater que, malgré le 
grand nombre de recherches et les démarches préventives mises en place ces dernières années afin de lutter contre le harcèlement, ce dernier reste un problème majeur et difficile à appréhender du fait de la part subjective. Afin d'améliorer le repérage du harcèlement dans un premier temps, nous avons traité de critères complémentaires concernant sa définition, en plus de la répétition, de la fréquence, de l'intensité et de l'asymétrie majoritairement retenues. Le harcèlement, comme processus relationnel violent, comprend des signes cliniques de souffrance aiguë qui révèlent souvent la présence de symptômes de stress post-traumatique et des traces, chez la victime, de mécanismes d'emprise, retrouvés dans toute violence psychologique. Nous avons également axé notre définition sur deux dimensions essentielles : l'adolescence et le groupe, et plus particulièrement sur la prise en compte de l'adolescence comme temps de fragilisation narcissique et de réactivation des problématiques psychiques, qui rend souvent nécessaire l'étayage sur un groupe de pairs, d'autant plus «vital » que ces jeunes s'éloignent parallèlement de leur groupe familial. Dans ces circonstances, certains adolescents, chez lesquels sont repérées des vulnérabilités familiales et psychologiques, éprouvant des difficultés à se positionner dans un groupe, peuvent devenir des proies «faciles » et faire l'objet de violences. Sans considérer un profil type de harcelé, la question des vulnérabilités psychologiques et notamment de la traumatophilie [30], soit l'appétence à la répétition de traumatismes et la surexposition à des situations potentiellement traumatiques, s'avère être une piste intéressante. Tous ces éléments nous indiquent la complexité du harcèlement qui tient en la superposition de facteurs familiaux et individuels ; mais aussi de reconnaître ce phénomène comme violence psychologique à part entière ayant des effets à long terme et la nécessité de prises en charge en charge spécifiques qui permettent, outre d'atténuer la souffrance en apportant un soutien, d'effectuer une analyse approfondie des enjeux psychiques sous-jacents à l'établissement d'une relation d'emprise et sa pérennisation. Le volet de la prévention secondaire, comportant des prises en charge spécifiques, au-delà des programmes de prévention primaire dans les établissements scolaires, nous a donc semblé important à développer 6 .

\section{Discussion avec l'auditoire}

\footnotetext{
${ }^{6}$ Une partie de ce projet « Dispositif thérapeutique double pour les adolescents victimes de harcèlement et leurs familles : théâtre-forum et groupe multifamilles » a obtenu un financement pour l'appel à projets « soutenir les jeunes en souffrance psychique» de la Fondation de France en décembre 2018 (numéro 00090960) ainsi qu'un deuxième financement pour l'appel à projets «fonds pour le civisme » par Facebook en février 2019 (en attente d'un numéro mais remise des prix visible sur Facebook).
} 
Mme H. Haliday - Vous dites qu'il n'y a pas de profil type - et cela m'a étonnée d'adolescent harcelé. Pourtant, vous dites aussi que le fait d'avoir été harcelé au primaire constitue un facteur de risque. Dans quelle mesure peut-on dire que le profil type d'un adolescent harcelé, c'est un élève de primaire harcelé ?

$\operatorname{Pr}$ A. Charles-Nicolas - Sans aller jusqu'au harcèlement ni même à la relation d'emprise, les simples moqueries font l'objet d'études qui relient la vulnérabilité anxieuse et dépressive à ces moqueries comme étiologie.

N. Chidiac - Pouvez-vous préciser la différence entre stalking et harcèlement que vous avez faite dans votre présentation?

Réponse du Rapporteur - à Mme Haliday : La présence d'un harcèlement au moment du collège est vraisemblablement liée à la présence d'un harcèlement dans l'enfance. Il renvoie alors le plus souvent à la notion de différence perçue par les enfants (couleur, handicap, particularité morphologique, port de signes remarquables [lunettes, coiffure] tout ce qui peut faire différence) et peut-être à des traits de construction de la relation à autrui de l'enfant harcelé.

Cependant, si l'on considère les derniers chiffres de l'Unicef, le harcèlement sous forme de moqueries répétitives est très fréquent en primaire (47 \% des enfants), dès lors peuton parler d'un profil type d'enfant harcelé ?

Enfin le lien harcèlement au primaire est lâche car les adolescents harcelés et en particulier ceux qui le sont par le cyber harcèlement sont dans une proportion importante aussi des enfants qui n'avaient pas subi de harcèlement antérieurement.

$\mathrm{Au}$ Pr Charles-Nicolas : oui tout à fait, les effets du harcèlement sont nombreux, durables et polymorphes dont des troubles anxieux et dépressifs, et pas forcément corrélés à l'intensité du harcèlement.

À Mme Chidiac : Selon la définition du clinicien américain John Reid Meloy, trois éléments permettent de définir le stalking :

- $\quad$ intrusion vis-à-vis d'autrui contre sa volonté ;

- $\quad$ menace implicite ou explicite manifestée dans le comportement de l'agresseur ;

- $\quad$ dont la conséquence est un sentiment de peur chez la victime.

Il s'agit d'une attitude compulsive d'un individu à l'égard d'un autre sans qu'il y ait eu relation antérieure. Si nous devions faire une différence nous pourrions dire que les situations de cyberharcèlement chez les jeunes sont le plus souvent le fait de personnes de l'entourage immédiat (ex-ami, groupe, classe). Le Stalking est issu d'une notion juridique servant à protéger les personnes d'intrusion abusive sur la toile pour compiler les éléments de vie d'une 
personne. Le harcèlement adolescent semble avoir plutôt comme ressort soit la différence comme en primaire, soit la déception, ou le rapprochement impossible.

Conflit d'intérêt : à compléter par l'auteur

\section{Références}

[1] Pepler D, Craig W, Jiang D, Connolly J. The development of bullying. International Journal of Adolescent Medicine and Health 2008;20:113- 19.

[2] Alvarez-Garcia D, Alvarez T, Carlos Nunez J. Predictors of school bullying perpetration in adolescence: A systematic review. Agression and violent behavior 2015;23:126-36.

[3] Cook C, et al. Predictors of childhood bullying and victimization. School Psychology Quarterly 2009;25:65-83.

[4] Olweus D. Bullying in schools: what we know and what we can do. London: Blackwell; 1993.

[5] Dorey R. La relation d'emprise. Nouvelle Revue de psychanalyse 1981;24: 1426, 117-40.

[6] Ferenczi S. (1932). Confusion des langues entre les adultes et l'enfant. Psychanalyse IV, Euvres complètes, Vol. 4. Paris: Payot; 1982: P. 274-87.

[7] Arnocky S, Vaillancourt T. A Multi-Informant longitudinal study on the relationship between aggression, peer victimization, and dating status in adolescence. Evolutionary Psychology 2012;10:253-70.

[8] Roques M, et al. Le harcèlement psychologique en milieu scolaire : une affaire de groupe d'adolescents? Effets traumatiques et propositions de prises en charge. Neuropsychiatrie de l'enfance et de l'adolescence 2015;63:533-40.

[9] Volk A, Craig W, Boyce W, King M. Adolescent risk correlates of bullying and different types of victimization. International Journal of Adolescent Medicine and Health 2006;18:37586.

[10] Reijntjes A, Vermande M, Olthof T, Goossens FA., Van de Schoot R, Tolman DL, McClelland SI. Normative sexuality development in adolescence: A decade in review, 20002009. Journal of Research on Adolescence 2011;21:242-55.

[11] Volk AA, Dane AV, Marini ZA, Vaillancourt T. Adolescent bullying, dating, and mating testing an evolutionary hypothesis. Evol Psychol 2015;13:1-11.

[12] White DD, Gallup AC, Gallup GG. Indirect peer aggression in adolescence and reproductive behavior. Evolutionary Psychology 2010;8:49-56.

[13] Arnocky S, Vaillancourt T. A Multi-Informant Longitudinal Study on the Relationship 
between Aggression, Peer Victimization, and Dating Status in Adolescence. Evolutionary Psychology 2012;10:253-70.

[14] Connolly J, Pepler D, Craig W, Taradash A. Dating experiences of bullies in early adolescence. Child Maltreatment 2000;5:299-31.

[15] Anzieu D, Martin JY. La dynamique des groupes restreints. Paris: PUF; 1968.

[16] Wolke D, Lereya ST. Long-term effects of bullying. Archives of Disease in Childhood 2015;100:879-85.

[17] Kaess M. Bullying: peer-to-peer maltreatment with severe consequences for child and adolescent mental health. European Child \& Adolescent Psychiatry 2018;27:945-7.

[18] Roques M, Hurvy C. Épreuves projectives et recherche en psychologie clinique. Paris: In Press; 2016.

[19] Corman L. Le test du dessin de la famille. Paris: PUF; 1970.

[20] Jourdan-Ionescu C, Lachance J. Le dessin de la famille. Paris: EAP; 2000.

[21] Royer J. Le dessin d'une maison. Image de l'adaptation sociale de l'enfant. Paris: Éditions et applications psychologiques; 1989.

[22] Hurvy C, Roques M. L'importance du domicile dans la réalité psychique - enjeux thérapeutiques en psychiatrie. Ann Méd Psychol 2018;176:518-22.

[23] Frost N. Qualitative Research Methods in Psychology: Combining Core Approaches: From core to combined approaches. Open University Press; 2011.

[24] Smith JA. Qualitative psychology: A practical guide to research methods. London: Sage. 3rd edition; 2015.

[25] Braun V, Clarke V. Using thematic analysis in psychology. Qualitative Research in Psychology 2006;3:77-101.

[26] Damiani C, Peireira-Fradin M. TraumaQ, questionnaire d'évaluation du traumatisme, Paris: ECPA; 2006.

[27] Dérogatis LR. SCL-90 R., Inventaire de symptômes psychologiques en autoquestionnaire. Paris: ECPA; 2015.

[28] Brun A, Chouvier B, Roussillon R. Manuel des médiations thérapeutiques. Paris: Dunod; 2019.

[29] Tisseron S. (2012). Prévenir la violence et le harcèlement scolaire. J Psychol 2013;299:28-32.

[30] Guillaumin J. Besoin de traumatisme et adolescence. Hypothèse psychanalytique sur une dimension cachée de l'instinct de vie. Adolescence 1985;3:127-37. 